\title{
Proteasome Binding
}

National Cancer Institute

\section{Source}

National Cancer Institute. Proteasome Binding. NCI Thesaurus. Code C41596.

Proteasome Binding involves temporary non-covalent interaction through intermolecular physical forces of attraction with multicatalytic macromolecular cellular structures in the cytosol and nucleus with multiple proteolytic activities involved in ATP-dependent ubiquitinated protein degradation and antigen processing. 